\title{
A Novel Color Reduction Based Image Segmentation Technique For Detection Of Cancerous Region in Breast Thermograms
}

\author{
Amin Habibi ${ }^{1,}$, , Mousa Shamsi ${ }^{2}$ \\ Department of Electrical Engineering, Faculty of Electrical Engineering Sahand university of Technology Tabriz, Iran
}

\begin{abstract}
Segmentation of an image into its components plays an important role in most of the image processing applications. In this article an important application of image processing in determination of Breast Cancer is studied, and A Novel Image Segmentation Technique is proposed in order to determine Cancer in Breast Thermograms. First, this image is converted from RGB to color space HSV. Then Breast shape is extracted by ACM algorithm. Finally, the image has segmented using Color Reduction Based algorithm. Experimental results on the acquired images show Accuracy of the proposed algorithm on the acquired images is over 90\% for healthy pixels and defected ones.

Keywords: Image Segmentation, Breast Cancer, Color Space, Breast Thermograms, Active Contour Model, Self Organized Map, Neural Networks
\end{abstract}




\section{Introduction}

B reast diseases are one of the major issues in women's health today. Early detection of breast cancer lays a significant role in reducing the mortality rate. By identifying and removing malignant tumors in early tages before they metastasize and spread to neighboring regions, cancer threats could be halted. Advances in infrared (IR) camera technologies, appropriate patient protocols and properly calibrated thermography have lead to an increased interest in the application of IR systems in the medical imaging especially in breast cancer detection. Breast thermography (figure1) with proper protocols [1] is a potential early detection method which is non-invasive, non-radiating, passive, fast, painless, low cost, risk free and zero contact with the body [2-7]. Breast thermography is suitable for women of all ages, including pregnant or nursing women, with all sizes of breast, with or without breast implants, fibrocystic breasts, and breast of dense tissue with younger age [8]. Blood vessel activity and heat indicate the presence of precancerous cells or cancer cells in their early stages of development. It can detect early signs of cancer 810 years before mammography does [9]. While in functional thermography, physiological changes are captured; in mammography, anatomical changes are detected. It is worth noting that physiological changes eventually would lead to anatomical changes. Cancerous cells generate more heat than normal cells due to their higher metabolic activity, their angiogenesis, and their vascular dilation [10-12]. Breast cancerous tumors influence the breast thermal patterns which contain important information about the goodness of health and type of the tumors $[13,14]$.

Breast thermograms can be processed using image processing methods to enhance regions such as hot spots, segment and align relevant portions. The resultant images exhibit hot spot pixel clusters that vary in shape, size, location and number and have different distribution characteristics for different classes of images such as malignant, benign and normal. These differences are often subtle and apparent only to a trained eye. Automated classification of breast thermograms using these images is of interest especially for mass screening application. This requires the use of feature extraction and classification techniques. It is desirable that features be robust to intra-class variations such as small amounts of translation, rotation, spatial scale and additive noise. Higher order spectral invariant features are suitable from this perspective.

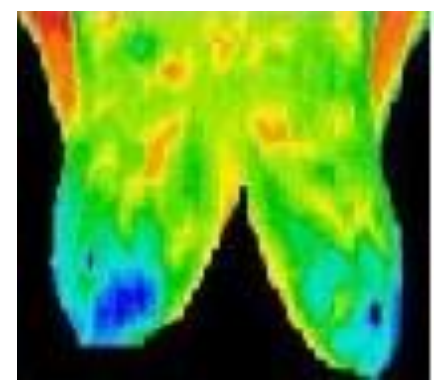

Fig. 1. Breast thermography

This paper is organized as follows: A brief literature review on hue-saturation-value (HSV) and the advantages of this color space to Rgb color space. Section 2 provides background on image processing techniques used in the processing of breast thermal images and on feature extraction from them and Introduce active countor model to extract the breast shape .Next we describe a mean of color reduction consider to Concept of self organized map a kind of neural networks and in section 4 to detect the cancer region exactly.

\section{Proposed Algorithm}

Figure 2 shows flowchart of the proposed algorithm. For color image segmentation converted from RGB to color space HSV. Because with survey of color histogram in each different color spaces, it is observed that the color space HSV provides better feature space for segmentation of color images than other color spaces. Then, the shape of breast image is extracted by active Contour model (ACM) algorithm 


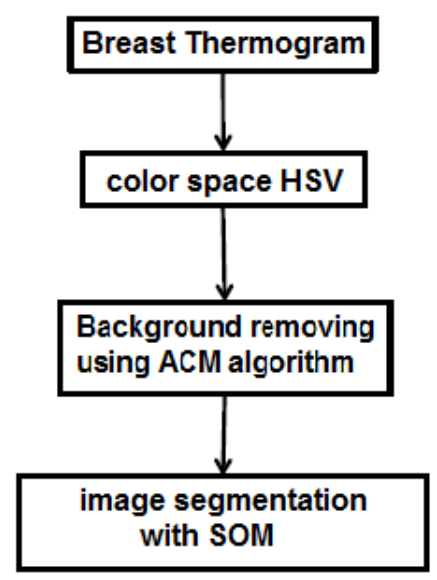

Fig. 2. flowchart of the proposed algorithm

\section{1. review on color space and hue- saturation-value (HSV)}

A color space defines a subset of colours for which a set of numbers used to describe the colour can be directly mapped to the manner in which the human eye responds to the colour.

In image processing and computer graphic, there are many coloring modes and models to represent the colors of the image, HSV(hue-saturation-value) color space is closer to human vision. Components of RGB color space have lighting and color properties together. Human vision is different to color and lighting. HSV help to proper identification of defects. so I have utilized HSV color space and it results good for image processing .

So, we convert from RGB to HSV in which Hue $(\mathrm{H})$ and Saturation $(\mathrm{S})$ contains the color information only and Value (V) contains the intensity information only. So, this channel of intensities can be processed alone without affecting the color information. After processing, color information can be again combined with the intensity information.

In all of these color spaces hue is defined the same way and is generally taken to be in the range of 0-359 with hue $=0$ being equivalent to hue $=360$. Hue can be visualized as an angle. In the equation below the values or $\mathrm{R}, \mathrm{G}, \mathrm{B}$ are normalized to 1 .

$$
H=\operatorname{accros} \frac{\frac{1}{2}(2 R-G-B)}{\sqrt{(R-G)^{2}-(R-B)(G-B)}}
$$

In HSV the saturation and value (the color summarizes uses this model) are calculated thus

$$
\begin{gathered}
S_{H S V}=\frac{\max (R, G, B)-\min (R, G, B)}{\max (R, G, B)} \\
V_{H S V}=\max (R, G, B)
\end{gathered}
$$

\subsection{Breast shape extraction using ACM algorithm}

Model-based approaches towards image interpretation named deformable models have proven very successful. Among the earliest and most well-known deformable models is the ACM known as snakes proposed by Kass[14]. We have used ACM for removing the background, because the precision of ACM outperforms simple thresholding methods especially when defects exist near the edges on breast image. Simple threshold classifies the pixels belonging to breast (foreground) when the intensity of the pixels for breast is equal to the intensity of the background. However, such error approaches to zero in ACM. We have utilized a primary contour whose size is similar to that of breast. It will speed up ACM thus leading to few iterations and high conversion rates. A snake is an energy minimizing parametric contour that deforms over a series of time steps. Each element along the contour $\mathrm{u}$ depends on two parameters, where the space parameter $\mathrm{s}$ is taken to vary between 0 and $\mathrm{N}-1$, and $\mathrm{t}$ is time (iteration):

$$
u(s, t)=(x(s, t), y(s, t))
$$

The total energy $E_{\text {snake }}$ of the model is calculated by the sum of the energy for the individual snake elements:

$$
E_{\text {snake }}=\int_{0}^{N-1} E_{\text {element }}(u(s, t)) d s
$$

The energy for each element can be decomposed into three basic energy terms: 


$$
E_{\text {element }}=E_{\text {int }}(u)+E_{\text {ext }}(u)+E_{\text {image }}(u)
$$

Each point on a contour moves adjacently in order to minimize the $\mathrm{E}$ snake in each step of the process of repetition.

The process stops when a local minimum is met .The internal energy (Einternal) regulates the constraints arranged on the model tension and stiffness. The external energy (Eexternal) is represented by external constraints im-posed by high-level sources such as human operators or automatic initialization procedures.

The image (potential) energy ( Eimag), drives the model towards the significant features, usually attributed by light and dark lines, edges or terminations[15].

The internal energy of a snake element is defined as :

$$
E_{\text {internal }}(u)=\alpha(s)\left|\frac{\delta u}{\delta s}\right|^{2}+\beta(s)\left|\frac{\delta^{2} u}{\delta^{2} s}\right|
$$

Here the first-order term is controlled by $\alpha(\mathrm{s})$, and the second-order term is controlled by $\beta(\mathrm{s})$. Minimizing the first-order energy term makes the snake contract by intro-ducing tension. Minimizing the second-order term makes the snake resist bending by producing stiffness. In other words, the curve is predisposed to have minimal (preferably zero) velocity and acceleration with respect to the parameter $\mathrm{s}$. The weights $\alpha(s)$ and $\beta(s)$ control the relative importance of the tension and stiffness terms.

Both manual and automatic supervision can be applied to control the external, driving the ACMs forcefully toward or away from a specific feature[15].

The potential (image) energy $\mathrm{P}$ is generated by image processing $\mathrm{I}(\mathrm{x}, \mathrm{y})$ in order to drive snakes towards the fea-tures. The energy parameters drive snakes towards lines (regions), edges and detecting termination (corners). The total image energy can be expressed as a weighted combination of these functions:

$$
p(u)=w_{\text {line }} \cdot E_{\text {line }}(u)+w_{\text {edge }} \cdot E_{\text {edge }}(u)+w_{\text {term }} \cdot E_{\text {term }}(u)
$$
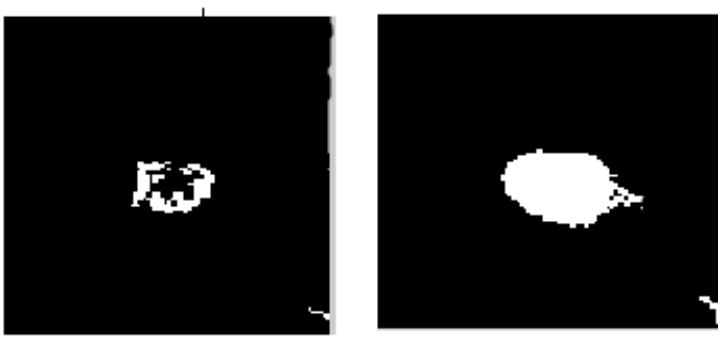

Fig. 4. manual segmentation of Cancerous Region in mammograms

I think Instead of manual segmentation that criteria of most Evaluation if we can Somehow this manual segmentation do with computer
The image energy is thus a linear combination of line, edge and termination energy terms, all computed from the raw image[15]. Figure 3 shows the $\mathrm{ACM}_{(6)}$ glgorithm result in different
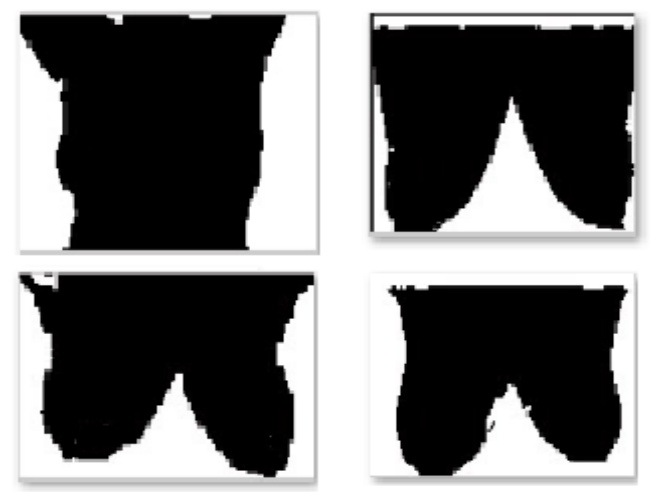

Fig. 3. ACM algorithm result in different iterations

\section{Color Reduction Idea}

In most defect detection problems, after segmentation an (Timage of focoused area for evaluation work results different ways used, one of them showed in figure 4, manual segmentation of area ( for example Cancerous Region in mammograms) and then compare that's with result image from segmentation of original image .
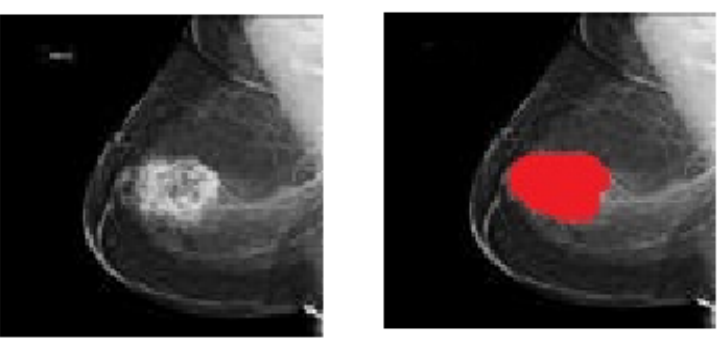
Certainly We'll be near to our purpose, Solution for it was color reduction[16] .

With color reduction in thermograms, somehow we segment a region we should be detect and that's desired goal for us to detect the Cancerous Region in breast thermograms, figure 5 .

\subsection{Color Reduction}

Color is one of the most important properties for object detection. Color Reduction of Image (CRI) is an important factor for segmentation, compression, presentation and transmission of images. The main purpose of CRI is to cut off the image storage spaces and computation time, An effective approach to solve CRI problem is to consider it as a clustering problem and solve it by using some adaptive clustering methods, such as SOM .

\subsection{SOM neural network}

SOM belongs to unsupervised learning of artificial neural networks, which has been widely used in clustering tasks, dimensionality reduction, data mining, information extraction, density approximation, data compression, etc [17]. A basic principle of unsupervised learning is the competition mechanism, in which the output units compete for activation. In some competitive learning algorithms only one output neuron is activated at any given time. This is realized by means of the so-called Winner-TakesAll (WTA) mode (e.g., LBG and K-means) or hard competitive learning. Another widely used mode is Winner-Takes-More (WTM), i.e., soft competitive learning [18]. The WTM mode is characterized by adapting in addition to the winner also some other units of the network. SOM adapt their weights all by using WTM mode or soft competitive learning.

CRI can be considered as the problem of transforming the original color image to a new one, which has only a few colors and nearly the same local characteristics. An effective approach to solve this problem is to consider it as a clustering problem and solve it by using some adaptive clustering methods, such as SOM network.

As we know, the main goal of SOM is the representation of a large set of input vectors with a smaller set of prototype vectors (or reference vectors), in order to get an approximation of the original input space to be obtained. In other words, a SOM neural network can decrease the input feature space into a smaller one. The resultant feature space (network output layer) can be viewed as a representative of the original feature space (network input signals), figure5. Therefore, it can preserve the main statistical characteristics of the input space and it has been widely used for intelligent image processing, such as image segmentation, compression, presentation and transmission, etc.

The resultant feature space (network output layer) can be viewed as a representative of the original feature space (network input signals). Therefore, SOM can preserve the main statistical characteristics of the input space and can be used for CRI,figure 6 .

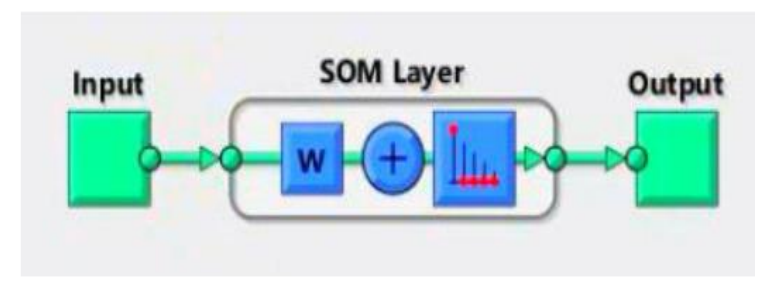

Fig. 5. Self Organized Map(SOM) Neural Network construction

The most often used CRI methods are based on nearest colors merging or color error diffusion. In the nearest color methods, each pixel in the image changes its color to the color in a palette that is the closest match to some typical neighboring pixels. A color palette is a fixed color table that has a limited number of colors. The error diffusion techniques belong to dithering approaches. The error refers to the cumulative difference between the actual values of pixels in the image and their "true" values. The two approaches have some limitations, for instance, the choice of the best colors is very difficulty. They are suitable for elimination of less frequently appeared colors in an image but are ineffective for image analysis and segmentation. , segmentation results of SOM algorithm shown in figure 7 anf figure 8 showed colores classification on reduced state. 


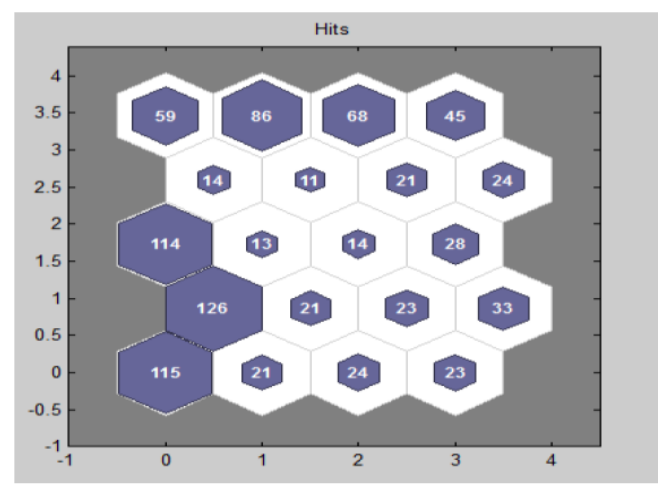

Fig. 6. Color classification with self organized map neural network
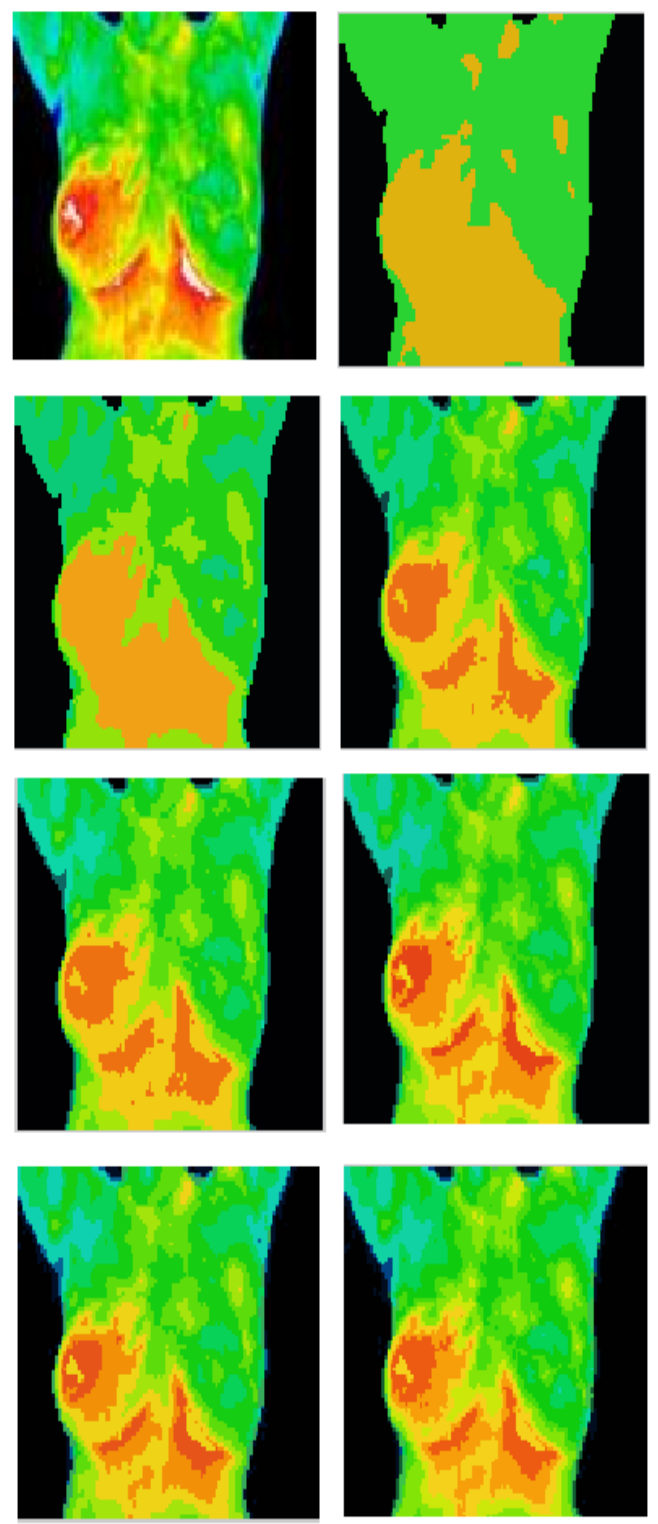

Fig. 7. Original image Color reduction results in different color numbers
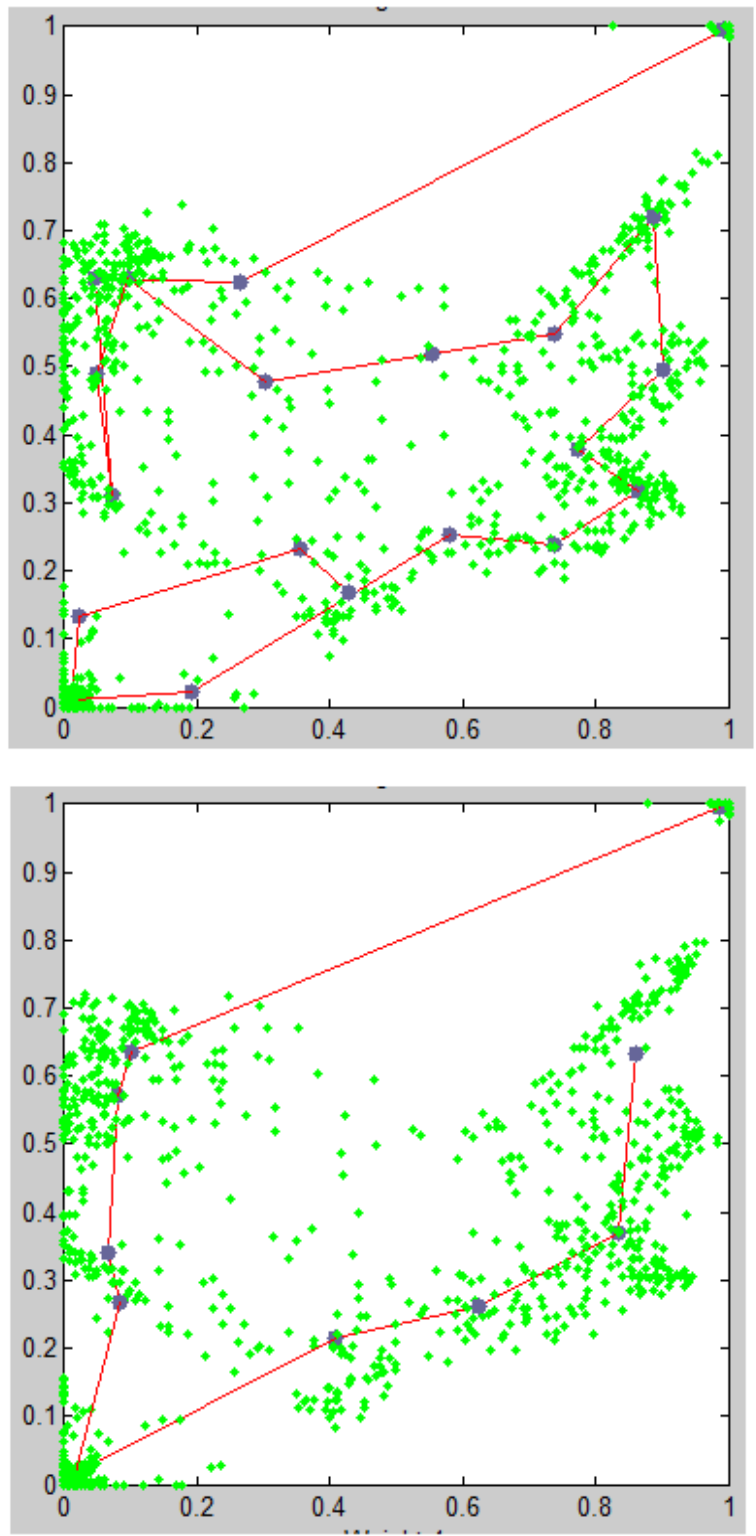

Fig. 8. Classification of colors in groups in different states

\section{Experimental Result}

Thermograms have a wide Range of colors and that's difficult to classification them to different groups for detect Similar regions, first algorithm applies to original thermograms to see results of them, results shows detect of desired areas from this images Almost impossible .

But, after color reduction of thermograms, algorithm succed, in detect of similar color regions. As mentioned above, thats is like to we segment manually thermograms and in this case diffirent regions are detectable with high examination, figure 9 . 

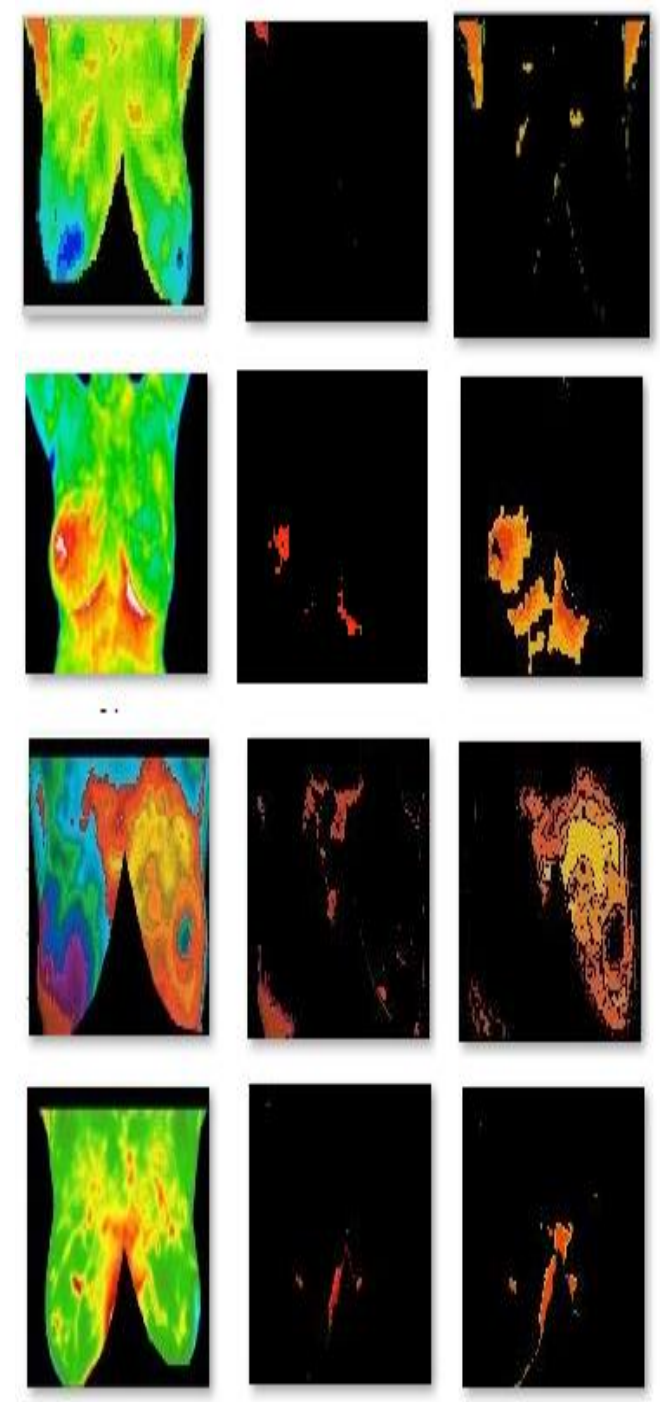

Fig. 9. The breast images segmentation result: First column: the original images, Second column: the results without color reduction, Third column: regimentation results with color reduction

\section{Conclusions}

In this paper, an important application of image process-ing in determination of Breast Cancer is mentioned and an algorithm is presented in order to determine Cancer in Breast Thermograms. The algorithm consists of three stages: First of all, the image was converted from RGB to color space HSV. Secondly, Breast shape was extracted using ACM algorithm. At last, in the third stage, the image is segmented by using SOM algorithm. Accuracy of the proposed algorithm on the acquired images was over $90 \%$ for healthy pixels and de-fected ones, respectively.The methods mentioned in intro- duction are of type supervised and have been applied on datasets which we do nothave access to. Moreover, our proposed method is of type unsupervised .

\section{References}

B.F. Jones, A reappraisal of the use of infrared thermal image analysis in medicine, IEEE Transactions on Medical Imaging 17 (1998) 10191027.

N. Diakides, J.D. Bronzino, Medical Infrared Imaging, CRC Press, Taylor \& Francis Group, 2008.

E.Y.K. Ng, A review of thermography as promising non-invasive detection modality for breast tumor, International Journal of Thermal Sciences 48 (2008) 849-855.

J.H. Tan, E.Y.K. Ng, U.R. Acharya, C. Chee, Infrared thermography on ocular surface temperature: a review, Infrared Physics \& Technology 52 (2009) 97-108.

E.Y.K. Ng, E.C. Kee, Advanced integrated technique in breast cancer thermography, Journal of Medical Engineering \& Technology 32 (2007) 103-114.

W.K. Ng, E.Y.K. Ng, Y.K. Tan, Qualitative study of sexual functioning in couple with ED: prospective evaluation of the thermography diagnostic system, The Journal of Reproductive Medicine 54 (2009) 698-705.

E.Y.K. Ng, G.J.L. Kaw, W.M. Chang, Analysis of IR thermal imager for mass blind fever screening, Microvascular Research 68 (2004) 104-109.

K.R. Foster, Thermographic detection of breast cancer, IEEE Engineering in Medicine and Biology Magazine 17 (1998) 10-14.

P.D. Keyserlingk, J.R. Ahlgren, E. Yu, N. Belliveau, Infrared imaging of breast: initial reappraisal using high-resolution digital technology in 100 successive cases of stage I and II breast cancer, The Breast Journal 4 (1998) 241-251. 
M. EtehadTavakol, C. Lucas, S. Sadri, E.Y.K. Ng, Analysis of breast thermography using fractal dimension to establish possible difference between malignant and benign patterns, Journal of Healthcare Engineering 1 (2010) 27-43.

H. Qi, P.T. Kuruganti, W.E. Snyder, Detecting breast cancer from thermal infrared images by asymmetry analysis, in: Biomedical Engineering Handbook, CRC, Boca Raton, 2006, pp. 27-1-2714.

L.G. Keith, J.J. Oleszczuk, M. Laguens, Circadian rhythm chaos: a new breast cancer marker, International Journal of Fertility and Women's Medicine 46(2001) 238-247.

J. Koay, C.H. Herry, M. Frize, Analysis of breast thermography with artificial neural network, in: 26th IEEE EMBS Conference, San Francisco, CA, USA, 2004, pp. 1159-1162.

M. Kass, et al., "Snakes: Active contour models," Interna-tional Journal of Computer Vision, vol. 1, pp. 321-331, 1988.

M. Nixon and A. Aguado, feature extraction and image processing, illustrated ed.: Newnes, 2002.

Guojian Cheng, Jinquan Yang, Kuisheng Wang, Xiaoxiao Wang, Image Color Reduction based on Self-organizing Maps and Growing SelfOrganizing Neural Networks, Sixth International Conference on Hybrid Intelligent Systems, 2-4/06 $\$ 20.00$ @ 2006

T. Kohonen, Self-Organizing Maps. Springer, Berlin, Heidelberg, 2001. (Third Extended Edition).

B. Fritzke, Some competitive learning methods. http://www.neuroinformatik..ruhr-unibochum.de/ini/VDM/research/gsn/JavaPaper/ 